\title{
Resultados auditivos posaticoantrostomía con antroexclusión
}

\section{Audiologic results post attico antrostomy with antrum exclusion}

\author{
Carlos Stott C1, Michel Royer F1', Lorena Ortúzar B², Marcela Pavón².
}

\begin{abstract}
RESUMEN
Introducción: Las intervenciones quirúrgicas del oído medio por colesteatoma tienen por objetivo prioritario la erradicación de la enfermedad, logrando en ocasiones conservar o mejorar la audición de estos pacientes. La aticotomía con antroexclusión es una cirugía conservadora que permite la eliminación de un colesteatoma atical manteniendo una anatomía cercana a la normal.

Objetivo: Este trabajo busca determinar los cambios auditivos en pacientes sometidos a este procedimiento quirúrgico.

Material y método: Se realiza un estudio transversal con audiometrías a pacientes sometidos a aticoantrostomía con antroexclusión entre los años 2000 y 2005. Se registran datos individuales, hallazgos intraoperatorios y valores audiométricos pre $y$ posoperatorios.

Resultados: Se incluyen 18 pacientes, edad promedio de 48 años, $61 \%$ de sexo femenino.

La diferencia ósea-aérea preoperatoria promedio es de $38 \mathrm{~dB}$ (10-51 $\mathrm{dB}$ ), mientras la posoperatoria es de $33 \mathrm{~dB}(18-50 \mathrm{~dB})$. El 50\% de los pacientes obtiene un cierre posoperatorio inferior a $30 \mathrm{~dB}$. No existen cambios significativos con respecto a umbrales de vías óseas o aéreas.

Conclusiones: La aticoantrostomía es un procedimiento quirúrgico que permite erradicar la enfermedad y preservar la audición de los pacientes. En nuestra serie existe una conservación de la audición, lo que constituye una ventaja con respecto a otras alternativas quirúrgicas.
\end{abstract}

Palabras clave: Colesteatoma, audición, aticotomía, antroexclusión

\begin{abstract}
Introduction. The primary aim of middle ear surgical intervention due to cholesteatoma is the elimination of the disease, while conserving or in some cases improving the patient's hearing ability. Atticostomy with antrum exclution is a conservative surgery that allows attic cholesteatoma removal, maintaining a relatively normal anatomy.
\end{abstract}

Médicos del Servicio de Otorrinolaringología, Hospital Clínico Universidad de Chile.

2 Interna de Medicina. Universidad de Chile. 
Aims. The main goal of this study is to characterize the audiological changes in patients that underwent this surgical procedure.

Material and methods. Audiometries were performed to patients that had atticostomy with antrum exclution, between years 2000 and 2005. Individual data, intrasurgery findings, and audiometric data prior and post surgery were recorded.

Results. Results from 18 patients, average age 48 years old, 61\% females, are presented. Pre surgical average difference between air and bone conduction was $38 d B$ (10-51dB), whereas post surgical difference was $33 d B$ (18-50). Fifty percent of patients reached a post surgical level lower than $30 \mathrm{~dB}$. There were no significant changes related to air or bone conduction thresholds.

Conclusions. Atticoantrostomy is a surgical procedure that allows both disease elimination and hearing preservation. In our series, hearing was preserved, which represents an advantage over other surgical alternatives.

Key words. Cholesteatoma, hearing, atticotomy, antrum exclusion.

\section{INTRODUCCIÓN}

El colesteatoma es una estructura de epitelio plano queratinizado ubicado en un sitio anómalo, siendo su localización más frecuente en el oído medio. Presenta un patrón de crecimiento independiente, reemplazando a la mucosa, con capacidad de reabsorción ósea y tendencia a recurrir tras su extracción ${ }^{1}$. La importancia de esta patología radica en su potencialidad para causar complicaciones severas, tales como meningitis, abscesos intracraneales, laberintitis y parálisis facial. En general es poco frecuente, con una prevalencia estimada de 3/100.000 en población pediátrica y 9/ 100.000 en adultos ${ }^{2}$.

El tratamiento definitivo del colesteatoma es únicamente quirúrgico. En su planificación se deben considerar tres objetivos: terapéutico, el erradicar la enfermedad como fin prioritario; anatómico, lograr una adecuada protección del oído frente a agentes externos; $y$ funcional, mantener o mejorar la capacidad auditiva, siendo estos últimos objetivos secundarios, pero recomendables en el tratamiento de esta enfermedad ${ }^{3,4}$.

Existen diversas técnicas quirúrgicas para la resección del colesteatoma, incluyendo mastoidectomía, epitimpanotomía y vaciamiento timpanomastoideo, con conservación o destrucción de la pared posterior. La aticoantrostomía con antroexclusión, descrita inicialmente por Olaizola ${ }^{5}$ se compone del abordaje del ático, eliminación de la pared lateral del ático, con 0 sin preservación de los cuerpos de martillo y yunque, la erradicación del colesteatoma y la reconstrucción de la pared posterior mediante un injerto de cartílago que se continua hasta el tegmen, cerrando el paso desde el oído medio al antro, dejando el ático abierto al conducto auditivo externo (Figura 1). Esta técnica

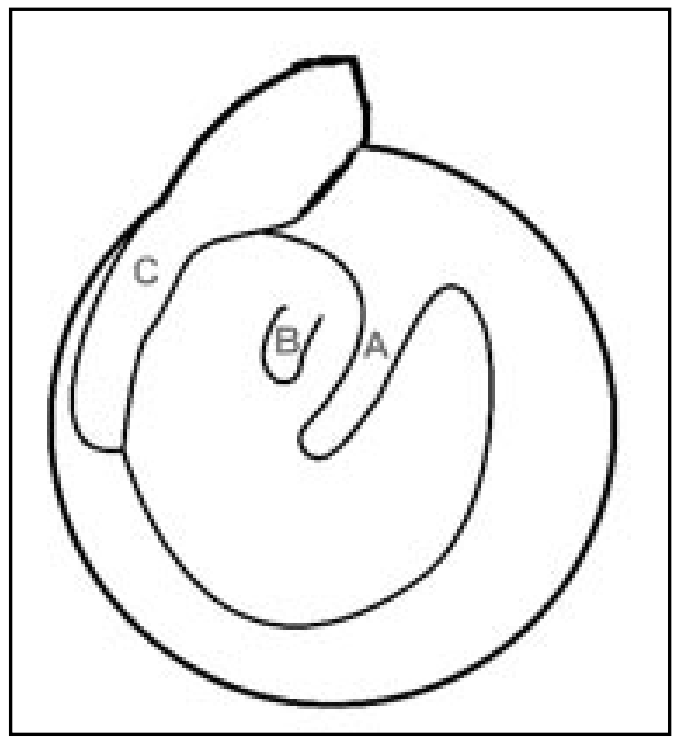

Figura 1. Esquema de reconstrucción pared posterior con cartílago tragal. A: Martillo; B: Articulación incudoestapedial; $C$ : cartílago tragal en pared posterior. 
permite eliminar la enfermedad y mantener una anatomía cercana a la normal. Conservar la estructura de la pared posterior del conducto auditivo externo permitiría conservar sus características acústicas, su relación con el mecanismo de conducción ${ }^{6,7}$; la vibración de la cadena osicular ${ }^{8}$ y el balance de presión del oído medio ${ }^{9}$. De esta forma se mantendría una adecuada vibración del tímpano y una caja timpánica ventilada, y el bloqueo del antro mastoideo lograría prevenir la retracción del remanente 0 del injerto timpánico, lo que se traduciría en una mejor funcionalidad en cuanto al nivel auditivo del paciente ${ }^{10,11}$.

En nuestro centro se realiza este procedimiento desde el año 2000, por lo que parece interesante determinar la audición postoperatoria en estos pacientes, dado que sería una de los beneficios de esta técnica quirúrgica.

\section{MATERIAL Y MÉTODO}

Se realizó un estudio transversal descriptivo, retrospectivo, durante el año 2006 incluyendo pacientes con diagnóstico de colesteatoma, a los cuales se les realizó aticoantrostomía con antroexclusión en el Hospital Clínico de la Universidad de Chile entre los años 2000 a 2005. Se consideraron candidatos a este procedimiento aquellos pacientes con colesteatoma presente en ático y additus ad antrum, sin compromiso del antro mastoídeo. Se excluyeron del análisis a pacientes con antecedentes incompletos en cuanto a audición preoperatoria y evento quirúrgico.

A cada paciente se le realiza una audiometría tonal en el Servicio de Otorrinolaringología. Se expresa el promedio de umbrales de tonos puros (PTP) en 500, 1000 y $2000 \mathrm{~Hz}$. para vías aérea, ósea y la diferencia ósea-aérea. Se revisan las fichas clínicas de cada paciente, registrándose los siguientes datos: identificación (edad, sexo), sintomatología predominante (hipoacusia, otorrea, vértigo, otalgia), antecedentes médicos, otomicroscopía, hallazgos en tomografía computada de oídos y nivel auditivo preoperatorio del paciente. Del protocolo operatorio se consignó el abordaje y los hallazgos quirúrgicos. Se destacan además la evolución clínica, presencia de complicaciones y/o recidiva.
La comparación de promedio de umbrales auditivos y diferencia ósea-aérea se realiza aplicando el test t de Student, considerando significativo un valor de $p$ menor a 0,05 .

\section{RESULTADOS}

Se incluyen en el estudio 18 pacientes con diagnóstico preoperatorio de colesteatoma, con edad promedio de 48 años (20 a 68 años). El $61 \%$ de ellos es de sexo femenino. La sintomatología predominante fue hipoacusia $(83,3 \%)$ y otorrea $(77,8 \%)$, siendo el vértigo y la otalgia considerablemente menores $(16,7 \%$ y $11,1 \%$ respectivamente). De los antecedentes médicos previos, sólo 3 pacientes refirieron episodios frecuentes de otitis medias agudas en la infancia.

La otomicroscopía del oído sano fue descrita en la mayoría normal, encontrándose retracción timpánica en 3 pacientes (16,7\%), miringoesclerosis en uno $(5,6 \%)$ y perforación timpánica en 1 paciente $(5,6 \%)$. En cuanto al oído enfermo, se evidenció un bolsillo de retracción posterosuperior en 12 pacientes (72\%), perforación timpánica en 4 y un paciente presentaba una atelectasia timpánica grado III de Sade. La tomografía computada de oídos demostraba velamiento atical en el 100\% de los pacientes y en el $27 \%$ (5 pacientes) presentaba extensión a caja timpánica.

Se utilizó abordaje endopreauricular en el $67 \%$ de los pacientes y retroauricular en el restante. Entre los hallazgos quirúrgicos, en el $94 \%$ de los pacientes se encontró colesteatoma en el ático, mientras en un paciente se evidenció tejido de granulación sin piel. En el $72 \%$ de los casos existía erosión de la cadena osicular, con extensión de la enfermedad medial a ella. La alteración más frecuente fue la amputación de la rama larga del yunque en el $27 \%$. La supraestructura del estribo se encontraba presente y con movilidad adecuada en el $44 \%$ de los casos. En el $67 \%$ de esta serie de pacientes se realizó osiculoplastía, en la que se utilizó cartílago tragal.

El 94\% de los pacientes fue dado de alta al día siguiente del procedimiento, mientras un paciente permaneció tres días hospitalizado debido a una crisis hipertensiva. No se presentaron casos de hipoacusia sensorioneural posoperatoria y en un 
paciente se produjo una paresia facial posoperatoria, transitoria y con recuperación completa.

En cuanto a los valores auditivos, el PTP preoperatorio para la vía aérea y el gap óseo-aéreo fue de 54,7 y $38 \mathrm{~dB}$, mientras en el posoperatorio disminuyen a 50,7 y $33 \mathrm{~dB}$ respectivamente (Figura 2). Estos cambios pre-posquirúrgicos no son estadísticamente significativos. El $50 \%$ de los pacientes obtuvieron un cierre postoperatorio del gap óseo-aéreo inferior a $30 \mathrm{~dB}$, distribuyéndose la mayoría entre 20 a $40 \mathrm{~dB}$ (Figura 3). Durante el período de seguimiento de los pacientes, con un promedio de 2 años (1-6 años), no se han presentado recidivas.

\section{DISCUSIÓN}

El colesteatoma es una patología de resolución quirúrgica, pero existen algunas controversias sobre el tipo de cirugía a utilizar. Algunos autores prefieren la timpanomastoidectomía cerrada (canal wall up) debido a que evita los problemas de cavidad y presenta una óptima funcionalidad; en tanto, otros abogan por una técnica abierta (canal wall down) aludiendo a que permite una mejor visualización en el acto quirúrgico, mejor erradicación de la enfermedad y disminución en la recurrencia $^{12,13}$. Por ello, la descripción de técnicas como timpanoplastía a demanda con antroex-

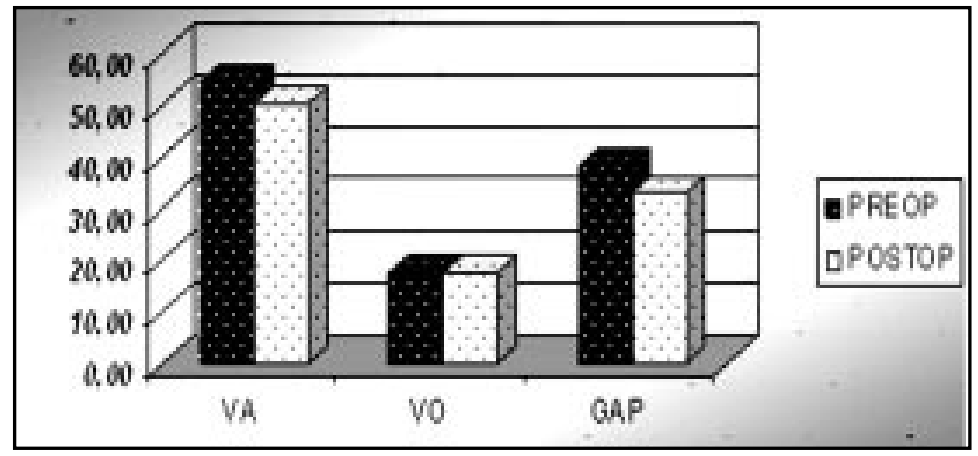

Figura 2. Valores audiométricos pre y posoperatorios de aticotomía con antroexclusión. VA: Umbral vía aérea; V0: Umbral vía ósea; GAP: Diferencia óseo-aérea.

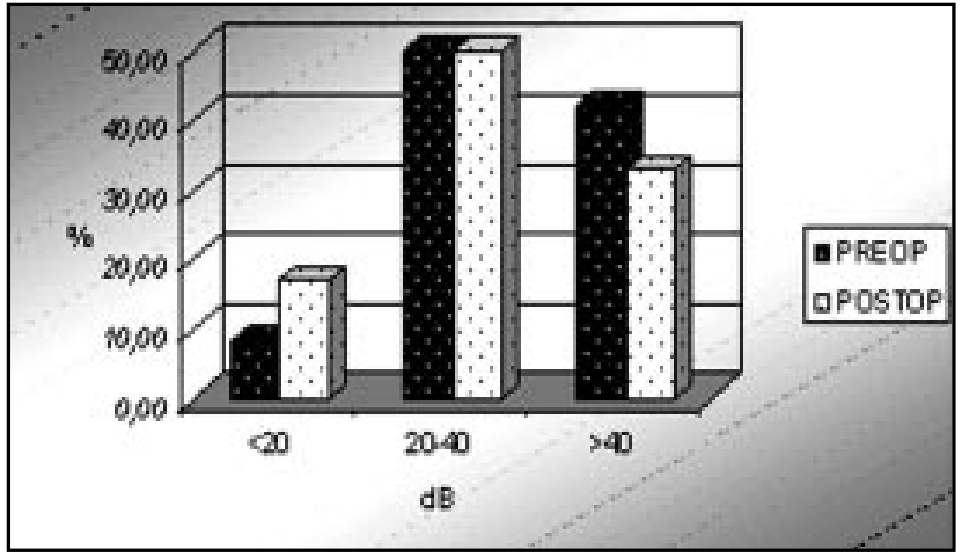

Figura 3. Diferencia ósea-áerea pre y posoperatoria de aticoticotomía con antroexclusión. 
clusión u osteoplastía posterior y atical ${ }^{14}$, buscan conjugar los beneficios de las técnicas cerrada y abierta en un procedimiento quirúrgico. De esta forma, se permite un abordaje adecuado de la zona afectada, se logra preservar la anatomía, se disminuye la posibilidad de recurrencia y se evitan los problemas relacionados con la cavidad (sobreinfección, estimulación térmica, etc...).

Preservar una anatomía cercana a la normal pretende mantener el rol del conducto auditivo externo sobre el mecanismo de conducción, y el bloqueo del antro mastoideo impide una gran cavidad que predisponga a la retracción timpánica. Estas ventajas funcionales pudieran representar un beneficio auditivo en estos pacientes, por lo que el objetivo de este trabajo fue determinar la variación de la audición posterior al procedimiento. En la mayoría de nuestros pacientes existe compromiso de la cadena osicular (72\%), siendo el hallazgo más frecuente la necrosis de la apófisis larga del yunque, lo que coincide con otras series ${ }^{15}$. La membrana timpánica fue reparada con fascia temporal y la osiculoplastía se realizó con injerto de cartílago tragal, lo que dificulta comparar los resultados con series internacionales en que se utilizan preferentemente prótesis de hidroxiapatita, las que no se encuentran disponibles de forma rutinaria en nuestro servicio. Si bien algunos autores ${ }^{16}$ no demuestran mayores beneficios con el uso de prótesis, conocer si en nuestras manos pueden representar una ventaja en las cirugías de oído medio será un desafío futuro.

En la presente serie se observa una conservación de la audición, lo que también se produce en otros estudios ${ }^{15}$. En cuanto a otras técnicas quirúrgicas, se ha descrito una similitud en sus resultados con la cirugía radical modificada, pero ésta mantiene problemas relacionados con la cavidad ${ }^{17}$. Además no hubo complicaciones posoperatorias relevantes, como hipoacusia sensorioneural severa o parálisis facial persistente y el promedio de días de hospitalización fue bajo.

Debe considerarse que esta técnica quirúrgica permite un abordaje adecuado al ático, especialmente anterior, y la cara posterior de la caja timpánica, pero no reemplaza a otras intervenciones frente a colesteatomas más extensos y/o asociados a complicaciones, por lo que debe enfatizarse una selección adecuada de los candidatos a este procedimiento.
Es destacable que ninguno de los pacientes haya presentado recidiva de colesteatoma. De todas formas debe considerarse que el tiempo de seguimiento en esta serie es variable y corto en general, describiéndose en el extranjero una frecuencia de recidiva entre $2,4 \%$ a $10 \%{ }^{15}$ para esta técnica, mejor que las cerradas y sólo superada por las técnicas abiertas ${ }^{13,18}$. La frecuencia real de recurrencia de la enfermedad en nuestros pacientes es un punto que deberá controlarse a futuro.

Ahora bien, un punto que llama la atención de esta técnica, es que sucede con el espacio aéreo de la mastoides, que ya no tiene comunicación con la caja timpánica. Podemos decir que no pasa nada, el aire sigue ahí, no produciéndose ninguna alteración tras ser excluida del resto del espacio ventilado por la trompa.

La otra interrogante es si las recidivas pudieran quedar ocultas por el cartílago que ocluye el additus. Creemos que no, ya que ese trozo de cartílago obstruye el antro y todo el resto de la caja queda visible transcanal, ya que la reparación de la membrana timpánica, cuando fue necesaria repararla, se realizó con fascia temporal y sólo se ocupó cartílago en la osiculoplastía.

En conclusión, la aticoantrostomía con antroexclusión es una alternativa quirúrgica frente a colesteatomas localizados, permitiendo un abordaje adecuado y evitando los inconvenientes posoperatorios con cavidades demasiado amplias. Permite conservar la audición de los pacientes y, además, es un procedimiento seguro, con baja frecuencia de complicaciones y recidivas. Por estos puntos parece apropiado incorporar esta técnica en el enfrentamiento de colesteatomas localizados en el oído medio.

\section{Agradecimientos}

Agradecimientos a los Tecnólogos Médicos de nuestro servicio Srs. Rubén Olmedo y Cristian Martínez.

\section{BIBLIOGRAFÍA}

1. Mc Cabe BF, Sade J, Abramson M. Summary Reports on National-International Conferences. First International Conference on Cholesteatoma. ANN Otol Rhinol Laryngol 1976; 85: 844-5. 
2. Olszewska E, Wagner M, Bernal-Sprekelsen M et AL. Etiopathogenesis of Cholesteatoma. Eur Arch Otorhinolaryngol 2004; 261: 6-24.

3. Lacosta JL, Infante JC, Pisón F. Consideraciones sobre la cirugía funcional del colesteatoma. I. Técnicas cerradas. Acta Otorrinolaring Esp 1997; 48: 115-20.

4. Tos M. Manual of middle ear surgery. Volume 2: Mastoid surgery and reconstructive procedures. New York: Thieme Medical Publishers, 1995.

5. Olaizola F. Nuestro criterio actual en la cirugía de las timpanoplastias. Acta Otorrinolaring Esp 1989; 40: 219-22.

6. Magnan J, Chays A, Gignac D et al. Reconstruction of the posterior canal wall: Long-term results. In: Charachon R, García-lbáñez E, eds. Long Term Results and Indications in Otology and Otoneurosurgery: Proceedings of the Politzer Society Conferences in Ibiza, 1989 and Courchevel, 1990. New York: Kugler Publications, 1991; 57-61.

7. ARs B. La partie tympanale de l'os temporal. Cahiers Otorhinolaryngol 1983; 6: 439-523.

8. Ars B, Decraemer W, Ars-Piret N. Tympanoossicular allografts: Morphology and physiology. Am J Otol 1987; 8: 148-54.

9. Ars B, Ars-PIREt N. Middle ear pressure balance under normal conditions: Specific role of the middle ear structure. Acta Otorhinolaryngol Belg 1994; 48:339-42.

10. Sadé J, Berco E, Fuchs C. Results of preservation of the posterior canal wall in cholesteatoma surgery as related to middle-ear aeration. $J$ Laryngol Otol 1986; 100: 1351-8.

11. OLAIZOLA F. Tympanoplasty: a 5-year review of results using the a la demanda (AAD.technique. Am J Otol 1988; 9: 318-22.

12. Kos MI, Castrillon R, Montandon P, Guyot JP. Anatomic and functional long-term results of canal wall-down mastoidectomy. Ann Otol Rhinol Laryngol. 2004; 113(11): 872-6.

13. Roden D, Honrubia VF, Wiet R. Outcome of residual cholesteatoma and hearing in mastoid surgery. J Otolaryngol 1996; 25(3): 178-81.

14. BABIGHIAN G. Posterior and attic wall osteoplasty: Hearing results and recurrence rates in cholesteatoma. Otol Neurotol 2002; 23: 14-7.

15. Vaamonde Lago P, Frade Gonzalz C, Castro Vilas $C$, Lecguga García R, Labelella Caballero T. Resultados de la timpanoplastía con Antroexclusión en el tratamiento del Colesteatoma de Oído Medio. Acta de Otorrinolaringol Esp 2001; 52: 9-13.

16. Cook JA, Krishnan S, Fagan PA. Hearing results following modified radical versus canal-up mastoidectomy. Ann Otol Rhinol Laryngol 1996; 105: 379-83.

17. Castro C, Frade C, Ordosgoitia H, Vélez MM, del Río M, LABELLA T. Resultados anatómicos y funcionales de la técnica de antroexclusión de Olaizola. Acta Otorrinolaringológica Gallega 1996; 3: 5-10.

18. Garzón JA. Nuestra experiencia en la cirugía reconstructiva del oído medio en timpanoplastias con mastoidectomía. Acta Otorrinolaring Esp 1994; 45: 315-27. 